\title{
Interesting Explicit Expressions of Determinants and Inverse Matrices for Foeplitz and Loeplitz Matrices
}

\author{
Zhaolin Jiang ${ }^{1}$, Weiping Wang ${ }^{1}$, Yanpeng Zheng ${ }^{2, *}$, Baishuai Zuo ${ }^{1}$ and Bei Niu ${ }^{1}$ \\ 1 School of Mathematics and Statistics, Linyi University, Linyi 276000, China; jiangzhaolin@lyu.edu.cn or \\ jzh1208@sina.com (Z.J.); weiping227@sina.cn (W.W.); bszuo124@163.com (B.Z.); \\ beiniu@stu.xidian.edu.cn (B.N.) \\ 2 School of Automation and Electrical Engineering, Linyi University, Linyi 276000, China \\ * Correspondence: zhengyanpeng@lyu.edu.cn
}

Received: 4 September 2019; Accepted: 6 October 2019; Published: 11 October 2019

\begin{abstract}
Foeplitz and Loeplitz matrices are Toeplitz matrices with entries being Fibonacci and Lucas numbers, respectively. In this paper, explicit expressions of determinants and inverse matrices of Foeplitz and Loeplitz matrices are studied. Specifically, the determinant of the $n \times n$ Foeplitz matrix is the $(n+1)$ th Fibonacci number, while the inverse matrix of the $n \times n$ Foeplitz matrix is sparse and can be expressed by the $n$th and the $(n+1)$ th Fibonacci number. Similarly, the determinant of the $n \times n$ Loeplitz matrix can be expressed by use of the $(n+1)$ th Lucas number, and the inverse matrix of the $n \times n(n>3)$ Loeplitz matrix can be expressed by only seven elements with each element being the explicit expressions of Lucas numbers. Finally, several numerical examples are illustrated to show the effectiveness of our new theoretical results.
\end{abstract}

Keywords: determinant; inverse; Fibonacci number; Lucas number; Toeplitz matrix; Hankel matrix

MSC: 15A09; 15A15; 15A69; 65F05

\section{Introduction}

Toeplitz matrices often arise in statistics, econometrics, psychometrics, structural engineering, multichannel filtering, reflection seismology, etc. (see [1,2] and references therein). Furthermore, they have been employed in quite wide fields of applications, especially in the elliptic Dirichlet-periodic boundary value problems [3], solving fractional diffusion equations [4-6], numerical analysis [7], signal processing [7], and system theory [7], etc. Citations of a large number of results have been made in a series of papers and in the monographs of Iohvidov [8] and Heining and Rost [9].

It seems to be an ideal research area and current topic of interest to specify inverses of Toeplitz matrices as well as the special Toeplitz matrices involving famous numbers as entries. Some scholars showed the explicit determinant and inverse of the special matrices involving famous numbers. The authors [10] proposed the invertibility of generalized Lucas skew circulant matrices and provided the determinant and the inverse matrix. Furthermore, the invertibility of generalized Lucas skew left circulant matrices was also discussed. The determinant and the inverse matrix of generalized Lucas skew left circulant matrices were obtained respectively. The determinants and inverses of Tribonacci skew circulant type matrices were discussed in [11]. The authors provided determinants and inverses of circulant matrices with Jacobsthal and Jacobsthal-Lucas numbers in [12]. The explicit determinants of circulant and left circulant matrices including Tribonacci numbers and generalized Lucas numbers were shown based on Tribonacci numbers and generalized Lucas numbers only in [13]. Moreover, four kinds of norms and bounds for the spread of these matrices were discussed respectively. In [14], circulant type matrices with the $k$-Fibonacci and $k$-Lucas numbers were considered and the 
explicit determinant and inverse matrix were presented by constructing the transformation matrices. Jiang et al. [15] gave the invertibility of circulant type matrices with the sum and product of Fibonacci and Lucas numbers and provided the determinants and the inverses of the these matrices. Jiang and Hong [16] studied exact form determinants of the RSFPLR circulant matrices and the RSLPFL circulant matrices involving Padovan, Perrin, Tribonacci, and the generalized Lucas number by the inverse factorization of a polynomial. It is worthwhile to note that Akbulak and Bozkurt gave the upper and lower bounds for the spectral norms of the Fibonacci and Lucas Toeplitz matrices [17].

In this paper, we will show the explicit determinants and inverses of the Foeplitz matrix and Fankel matrix both involving Fibonacci numbers (see Definitions 1 and 2 below), and the Loeplitz matrix and Lankel matrix both involving Lucas numbers (see Definitions 3 and 4). The main results are obtained by factoring the considered matrices into structured factors, whose determinant and inverse are computed exactly, and then reassembling the factorization. This paper provides a novel characterization of Fibonacci or Lucas numbers as the determinant of Toeplitz matrices containing numbers from the same sequence. In fact, the main contribution of this paper is that Toeplitz matrix, tridiagonal Toeplitz matrices with perturbed corner entries, the Fibonacci number, and the Golden Ratio are connected together.

Here the Fibonacci and Lucas sequences (see, e.g., [18]) are defined by the following recurrence relations, respectively:

$$
\begin{aligned}
& F_{n+1}=F_{n}+F_{n-1}(n \geqslant 1), \quad \text { where } \quad F_{0}=0, F_{1}=1, \\
& L_{n+1}=L_{n}+L_{n-1}(n \geqslant 1), \quad \text { where } \quad L_{0}=2, L_{1}=1, \\
& F_{-(n+1)}=-F_{-n}+F_{-(n-1)}(n \geqslant 1), \quad \text { where } \quad F_{0}=0, F_{-1}=1, \\
& L_{-(n+1)}=-L_{-n}+L_{-(n-1)}(n \geqslant 1), \quad \text { where } \quad L_{0}=2, L_{-1}=-1 .
\end{aligned}
$$

The following identities are easily attainable

$$
\begin{aligned}
& F_{-n}=(-1)^{n+1} F_{n}, L_{-n}=(-1)^{n} L_{n}, \\
& \sum_{i=2}^{n-2} a^{i} L_{k+i}=\frac{-a^{3} L_{k+1}-a^{2} L_{k+2}+a^{n-1} L_{n-1+k}+a^{n} L_{n-2+k}}{a^{2}+a-1}, a \neq \frac{-1 \pm \sqrt{5}}{2}, \\
& \sum_{i=2}^{n-2} a^{i} L_{k-i}=\frac{-a^{2} L_{k-2}-a^{3} L_{k-1}+a^{n-1} L_{k-(n-4)}+a^{n} L_{k-(n-2)}}{a^{2}-a-1}, a \neq \frac{1 \pm \sqrt{5}}{2} .
\end{aligned}
$$

Definition 1. An $n \times n$ Foeplitz matrix is defined as a Toeplitz matrix of the form

$$
T_{F, n}=\left(\begin{array}{ccccc}
F_{1} & F_{2} & \cdots & F_{n-1} & F_{n} \\
F_{-2} & F_{1} & \ddots & \ddots & F_{n-1} \\
\vdots & \ddots & \ddots & \ddots & \vdots \\
F_{-n+1} & \ddots & \ddots & F_{1} & F_{2} \\
F_{-n} & F_{-n+1} & \cdots & F_{-2} & F_{1}
\end{array}\right)_{n \times n}
$$

where $F_{1}, F_{ \pm 2}, \cdots, F_{ \pm n}$ are the Fibonacci numbers. 
Definition 2. An $n \times n$ Fankel matrix is defined as a Hankel matrix of the form

$$
H_{F, n}=\left(\begin{array}{ccccc}
F_{n} & F_{n-1} & \cdots & F_{2} & F_{1} \\
F_{n-1} & . \cdot & . \cdot & F_{1} & F_{-2} \\
\vdots & . \cdot & . \cdot & . \cdot & \vdots \\
F_{2} & F_{1} & . \cdot & . \cdot & F_{-n+1} \\
F_{1} & F_{-2} & \cdots & F_{-n+1} & F_{-n}
\end{array}\right)_{n \times n}
$$

where $F_{1}, F_{ \pm 2}, \cdots, F_{ \pm n}$ are the Fibonacci numbers.

Definition 3. An $n \times n$ Loeplitz matrix is defined as a Toeplitz matrix of the form

$$
T_{L, n}=\left(\begin{array}{ccccc}
L_{1} & L_{2} & \cdots & L_{n-1} & L_{n} \\
L_{-2} & L_{1} & \ddots & \ddots & L_{n-1} \\
\vdots & \ddots & \ddots & \ddots & \vdots \\
L_{-n+1} & \ddots & \ddots & L_{1} & L_{2} \\
L_{-n} & L_{-n+1} & \cdots & L_{-2} & L_{1}
\end{array}\right)_{n \times n}
$$

where $L_{1}, L_{ \pm 2}, \cdots, L_{ \pm n}$ are the Lucas numbers.

Definition 4. An $n \times n$ Lankel matrix is defined as a Hankel matrix of the form

$$
H_{L, n}=\left(\begin{array}{ccccc}
L_{n} & L_{n-1} & \cdots & L_{2} & L_{1} \\
L_{n-1} & . \cdot & . \cdot & L_{1} & L_{-2} \\
\vdots & . \cdot & . \cdot & . \cdot & \vdots \\
L_{2} & L_{1} & . \cdot & . \cdot & L_{-n+1} \\
L_{1} & L_{-2} & \cdots & L_{-n+1} & L_{-n}
\end{array}\right)_{n \times n}
$$

where $L_{1}, L_{ \pm 2}, \cdots, L_{ \pm n}$ are the Lucas numbers.

It is easy to check that

$$
\begin{aligned}
& H_{F, n}=T_{F, n} \hat{I}_{n}, \\
& H_{L, n}=T_{L, n} \hat{I}_{n},
\end{aligned}
$$

where $\hat{I}_{n}$ is the counteridentity matrix, the square matrix whose elements are all equal to zero except those on the counter-diagonal, which are all equal to 1 , which provide us with basic relations between $T_{F, n}$ and $H_{F, n}$, and $T_{L, n}$ and $H_{L, n}$, respectively. 
Lemma 1. ([19], Lemma 2.5) Define an $n \times n$ bi-band-Toeplitz matrix by

$$
\mathcal{F}_{n}(\alpha, \beta)=\left(\begin{array}{cccccc}
\alpha & 0 & \cdots & \cdots & \cdots & 0 \\
\beta & \alpha & \ddots & & & \vdots \\
0 & \beta & \alpha & \ddots & & \vdots \\
\vdots & \ddots & \ddots & \ddots & \ddots & \vdots \\
\vdots & & \ddots & \beta & \alpha & 0 \\
0 & \cdots & \cdots & 0 & \beta & \alpha
\end{array}\right)_{n \times n}
$$

the inverse of $\mathcal{F}_{n}(\alpha, \beta)$ can be expressed as

$$
\mathcal{F}_{n}(\alpha, \beta)^{-1}=\left(\begin{array}{ccccccc}
\Delta_{1} & 0 & \cdots & \cdots & \cdots & \cdots & 0 \\
\Delta_{2} & \Delta_{1} & \ddots & & & & \vdots \\
\Delta_{3} & \Delta_{2} & \Delta_{1} & \ddots & & & \vdots \\
\vdots & \ddots & \ddots & \ddots & \ddots & & \vdots \\
\Delta_{n-2} & \ddots & \ddots & \ddots & \ddots & \ddots & \vdots \\
\Delta_{n-1} & \Delta_{n-2} & \ddots & \ddots & \Delta_{2} & \Delta_{1} & 0 \\
\Delta_{n} & \Delta_{n-1} & \Delta_{n-2} & \cdots & \Delta_{3} & \Delta_{2} & \Delta_{1}
\end{array}\right)_{n \times n},
$$

where

$$
\Delta_{i}=\frac{(-\beta)^{i-1}}{\alpha^{i}}, i \geq 1 .
$$

Remark 1. This Lemma is a special case of ([19], Lemma 2.5).

\section{The Determinant and Inverse Matrix of Foeplitz, Fankel, Loeplitz, and Lankel Matrices}

In this section, we study the determinant and the inverse of Foeplitz, Fankel, Loeplitz, and Lankel matrices by factoring the considered matrices into structured factors, whose determinant and inverse are computed exactly, and then reassembling the factorization. We establish the relationship between the determinant of these matrices and Fibonacci or Lucas numbers.

\subsection{Determinant and Inverse Matrix of a Foeplitz Matrix}

In this subsection, the determinant and the inverse of the Foeplitz matrix $T_{F, n}$ are studied.

Theorem 1. Let $T_{F, n}$ be an $n \times n$ Foeplitz matrix defined as in (4). Then $T_{F, n}$ is invertible and

$$
\operatorname{det} T_{F, n}=F_{n+1}
$$

where $F_{n+1}$ is the $(n+1)$ th Fibonacci number. 
Proof. For $n \leq 3$, it is easy to check that $\operatorname{det} T_{F, 1}=1=F_{2}$, $\operatorname{det} T_{F, 2}=2=F_{3}$ and $\operatorname{det} T_{F, 3}=3=$ $F_{4}$. Therefore, Equation (10) is satisfied. Now, we consider the case $n>3$. Define two additional nonsingular matrices,

$$
A_{1}=\left(\begin{array}{cccccc}
1 & & & & & \\
-F_{-n} & & & & & 1 \\
-F_{-n-1} & & & 1 & -1 \\
0 & & & 1 & -1 & -1 \\
\vdots & & . & . & . \cdot & \\
0 & 1 & -1 & -1 & &
\end{array}\right)_{n \times n}, B_{1}=\left(\begin{array}{ccccc}
1 & 0 & \cdots & \cdots & 0 \\
0 & & & . & 1 \\
\vdots & & . & 1 & 0 \\
\vdots & . & . & . & \vdots \\
0 & 1 & 0 & \cdots & 0
\end{array}\right)_{n \times n}
$$

Multiplying $T_{F, n}$ by $A_{1}$ from the left, we obtain

$$
A_{1} T_{F, n}=\left(\begin{array}{cccccc}
F_{1} & F_{2} & F_{3} & \cdots & F_{n-1} & F_{n} \\
0 & \alpha_{2} & \alpha_{3} & \cdots & \alpha_{n-1} & \alpha_{n} \\
\vdots & \beta_{2} & \beta_{3} & \cdots & \beta_{n-1} & \beta_{n} \\
\vdots & 0 & \cdots & 0 & 1 & 0 \\
\vdots & \vdots & . & . & . & \vdots \\
0 & 0 & 1 & 0 & \cdots & 0
\end{array}\right)_{n \times n}
$$

where

$$
\begin{aligned}
& \alpha_{i}=-F_{-n} F_{i}+F_{-n+i-1},(i=2,3, \cdots, n), \\
& \beta_{i}=-F_{-n-1} F_{i}+F_{-n+i-2},(i=2,3, \cdots, n-1), \\
& \beta_{n}=-F_{-n-1} F_{n} .
\end{aligned}
$$

Then, multiplying $A_{1} T_{F, n}$ by $B_{1}$ from the right, we have

$$
A_{1} T_{F, n} B_{1}=\left(\begin{array}{cccccc}
F_{1} & F_{n} & F_{n-1} & \cdots & F_{3} & F_{2} \\
0 & \alpha_{n} & \alpha_{n-1} & \cdots & \alpha_{3} & \alpha_{2} \\
0 & \beta_{n} & \beta_{n-1} & \cdots & \beta_{3} & \beta_{2} \\
0 & 0 & 1 & 0 & \cdots & 0 \\
\vdots & \vdots & \ddots & \ddots & \ddots & \vdots \\
0 & 0 & \cdots & 0 & 1 & 0
\end{array}\right)_{n \times n}
$$

and

$$
\begin{aligned}
\operatorname{det}\left(A_{1} T_{F, n} B_{1}\right) & =\operatorname{det}\left(A_{1}\right) \operatorname{det}\left(T_{F, n}\right) \operatorname{det}\left(B_{1}\right) \\
& =F_{1}\left[(-1)^{n-1} \beta_{2} \alpha_{n}-(-1)^{n-1} \alpha_{2} \beta_{n}\right] \\
& =(-1)^{n-1} F_{1}\left[\left(-F_{-n} F_{n}+F_{1}\right)\left(-F_{-n-1} F_{2}+F_{-n}\right)+F_{-n-1} F_{n}\left(-F_{-n} F_{2}+F_{-n+1}\right)\right] .
\end{aligned}
$$

From the definition of $A_{1}$ and $B_{1}$, we get

$$
\operatorname{det} A_{1}=\operatorname{det} B_{1}=(-1)^{\frac{(n-1)(n-2)}{2}} .
$$


Therefore, we have

$$
\begin{aligned}
\operatorname{det} T_{F, n}= & (-1)^{n-1} F_{1}\left[\left(-F_{-n} F_{n}+F_{1}\right)\left(-F_{-n-1} F_{2}+F_{-n}\right)\right. \\
& \left.+F_{-n-1} F_{n}\left(-F_{-n} F_{2}+F_{-n+1}\right)\right] \\
= & F_{n+1}
\end{aligned}
$$

Since $F_{n+1} \neq 0$, the $n \times n$ Foeplitz matrix is invertible. Thus, the proof is completed.

Remark 2. Theorem 1 gives the relationship between the Foeplitz matrix and the Fibonacci number. From the perspective of number theory, the $(n+1)$ th Fibonacci number can be represented by the determinant of an $n \times n$ Foeplitz matrix.

Theorem 2. Let $T_{F, n}$ be an $n \times n$ Foeplitz matrix defined as in (4). The inverse matrix of $T_{F, n}$ is

$$
T_{F, n}^{-1}=\left(\begin{array}{cccccccccc}
\frac{F_{n}}{F_{n+1}} & -1 & 0 & 0 & 0 & \cdots & \cdots & \cdots & 0 & \frac{(-1)^{n}}{F_{n+1}} \\
1 & -1 & -1 & 0 & 0 & \cdots & \cdots & \cdots & 0 & 0 \\
0 & 1 & -1 & -1 & 0 & & & & & 0 \\
\vdots & \ddots & 1 & -1 & -1 & \ddots & & & & \vdots \\
\vdots & & \ddots & \ddots & \ddots & \ddots & \ddots & & & \vdots \\
\vdots & & & \ddots & \ddots & \ddots & \ddots & \ddots & & \vdots \\
\vdots & & & & \ddots & \ddots & \ddots & \ddots & \ddots & \vdots \\
\vdots & & & & & \ddots & 1 & -1 & -1 & 0 \\
0 & \cdots & \cdots & \cdots & \cdots & \cdots & 0 & 1 & -1 & -1 \\
-\frac{1}{F_{n+1}} & 0 & \cdots & \cdots & \cdots & \cdots & \cdots & 0 & 1 & \frac{F_{n}}{F_{n+1}}
\end{array}\right)_{n \times n}
$$

where $F_{n}$ and $F_{n+1}$ are the $n$th and $(n+1)$ th Fibonacci numbers, respectively.

Proof. For $n=1$, it is easy to check that

$$
T_{F, 1}=1 \text { and } T_{F, 1}^{-1}=\frac{F_{1}}{F_{2}} .
$$

For $n=2$, we have

$$
T_{F, 2}=\left(\begin{array}{cc}
1 & 1 \\
-1 & 1
\end{array}\right) \text { and } T_{F, 2}^{-1}=\left(\begin{array}{cc}
\frac{1}{2} & -\frac{1}{2} \\
\frac{1}{2} & \frac{1}{2}
\end{array}\right)
$$

and for $n=3$, we have

$$
T_{F, 3}=\left(\begin{array}{ccc}
1 & 1 & 2 \\
-1 & 1 & 1 \\
2 & -1 & 1
\end{array}\right) \text { and } T_{F, 3}^{-1}=\left(\begin{array}{ccc}
\frac{2}{3} & -1 & -\frac{1}{3} \\
1 & -1 & -1 \\
-\frac{1}{3} & 1 & \frac{2}{3}
\end{array}\right)
$$


which are in agreement with Equation (14). Now, we consider the case $n \geq 4$. The explicit expression of the inverse of the Foeplitz matrix can be obtained by use of Equation (14). Define addtionally two nonsigular matrices

$$
A_{2}=\left(\begin{array}{ccccccc}
1 & 0 & \cdots & \cdots & \cdots & \cdots & 0 \\
0 & 1 & \ddots & & & & \vdots \\
0 & -\frac{\beta_{n}}{\alpha_{n}} & 1 & \ddots & & & \vdots \\
\vdots & \ddots & 0 & \ddots & \ddots & & \vdots \\
\vdots & & \ddots & \ddots & \ddots & \ddots & \vdots \\
\vdots & & & \ddots & \ddots & 1 & 0 \\
0 & \cdots & \cdots & \cdots & 0 & 0 & 1
\end{array}\right)_{n \times n}
$$

and

$$
B_{2}=\left(\begin{array}{cccccc}
1 & -\frac{F_{n}}{F_{1}} & \frac{F_{n} \alpha_{n-1}}{\alpha_{n}}-F_{n-1} & \cdots & \frac{F_{n} \alpha_{3}}{\alpha_{n}}-F_{3} & \frac{F_{n} \alpha_{2}}{\alpha_{n}}-F_{2} \\
0 & 1 & -\frac{\alpha_{n-1}}{\alpha_{n}} & \cdots & -\frac{\alpha_{3}}{\alpha_{n}} & -\frac{\alpha_{2}}{\alpha_{n}} \\
\vdots & \ddots & 1 & 0 & \cdots & 0 \\
\vdots & & \ddots & \ddots & \ddots & \vdots \\
\vdots & & & \ddots & 1 & 0 \\
0 & \cdots & \ldots & \cdots & 0 & 1
\end{array}\right)_{n \times n},
$$

where $\alpha_{i}$ and $\beta_{n}$ are defined as in (11) and (12), respectively.

Multiplying $A_{1} T_{F, n} B_{1}$ by $A_{2}$ from the left and by $B_{2}$ from the right, we obtain

$$
\begin{aligned}
A T_{F, n} B & =A_{2} A_{1} T_{F, n} B_{1} B_{2} \\
& =\left(\begin{array}{ccccccc}
F_{1} & 0 & 0 & 0 & \cdots & 0 & 0 \\
0 & \alpha_{n} & 0 & 0 & \cdots & 0 & 0 \\
0 & 0 & \beta_{n-1}-\frac{\beta_{n} \alpha_{n-1}}{\alpha_{n}} & \beta_{n-2}-\frac{\beta_{n} \alpha_{n-2}}{\alpha_{n}} & \cdots & \beta_{3}-\frac{\beta_{n} \alpha_{3}}{\alpha_{n}} & \beta_{2}-\frac{\beta_{n} \alpha_{2}}{\alpha_{n}} \\
\vdots & \ddots & 1 & 0 & \cdots & \cdots & 0 \\
\vdots & & \ddots & 1 & \ddots & & \vdots \\
\vdots & & & \ddots & \ddots & \ddots & \vdots \\
0 & \cdots & \cdots & \cdots & 0 & 1 & 0
\end{array}\right)_{n \times n},
\end{aligned}
$$

where

$$
\begin{aligned}
& A=A_{2} A_{1}=\left(\begin{array}{ccccccc}
1 & & & & & 0 \\
-F_{n} & & & & & 1 \\
\frac{\beta_{n} F_{-n}}{\alpha_{n}}-F_{-n-1} & & & & 1 & -\frac{\beta_{n}}{\alpha_{n}}-1 \\
0 & & & 1 & -1 & -1 \\
\vdots & & . & . \cdot & . \cdot & \\
0 & 1 & -1 & -1 & &
\end{array}\right)_{n \times n}, \\
& B=B_{1} B_{2}=\left(\begin{array}{cccccc}
1 & -\frac{F_{n}}{F_{1}} & \frac{F_{n} \alpha_{n-1}}{\alpha_{n}}-F_{n-1} & \cdots & \frac{F_{n} \alpha_{3}}{\alpha_{n}}-F_{3} & \frac{F_{n} \alpha_{2}}{\alpha_{n}}-F_{2} \\
0 & \cdots & \ldots & \cdots & 0 & 1 \\
\vdots & & & . \cdot & 1 & 0 \\
\vdots & & . & . \cdot & . & \vdots \\
\vdots & . & 1 & 0 & \ldots & 0 \\
0 & 1 & -\frac{\alpha_{n-1}}{\alpha_{n}} & \cdots & -\frac{\alpha_{3}}{\alpha_{n}} & -\frac{\alpha_{2}}{\alpha_{n}}
\end{array}\right)_{n \times n} \text {, }
\end{aligned}
$$


with $\alpha_{i}$ and $\beta_{n}$ are defined as in (11) and (12), respectively. In addition, the matrix $A T_{F, n} B$ admits a block partition of the form

$$
A T_{F, n} B=N \oplus M,
$$

where $N \oplus M$ denotes the direct sum of the matrices $N$ and $M, N=\operatorname{diag}\left(F_{1}, \alpha_{n}\right)$ is a nonsingular diagonal matrix, and

$$
M=\left(\begin{array}{ccccc}
-\frac{\beta_{n} \alpha_{n-1}}{\alpha_{n}}+\beta_{n-1} & -\frac{\beta_{n} \alpha_{n-2}}{\alpha_{n}}+\beta_{n-2} & \cdots & -\frac{\beta_{n} \alpha_{3}}{\alpha_{n}}+\beta_{3} & -\frac{\beta_{n} \alpha_{2}}{\alpha_{n}}+\beta_{2} \\
1 & 0 & \cdots & \cdots & 0 \\
0 & 1 & \ddots & & \vdots \\
\vdots & \ddots & \ddots & \ddots & \vdots \\
0 & \cdots & 0 & 1 & 0
\end{array}\right)_{(n-2) \times(n-2)}
$$

From (15), we obtain

$$
T_{F, n}^{-1}=B\left(N^{-1} \oplus M^{-1}\right) A .
$$

Based on the defintions of $N$ and $M$, we have $N^{-1}=\operatorname{diag}\left(F_{1}^{-1}, \alpha_{n}^{-1}\right)$ and

$$
M^{-1}=\left(\begin{array}{ccccc}
0 & 1 & 0 & \cdots & 0 \\
\vdots & \ddots & \ddots & \ddots & \vdots \\
\vdots & & \ddots & \ddots & 0 \\
0 & \cdots & \cdots & 0 & 1 \\
\frac{\alpha_{n}}{\beta_{2} \alpha_{n}-\beta_{n} \alpha_{2}} & -\frac{\beta_{n-1} \alpha_{n}-\beta_{n} \alpha_{n-1}}{\beta_{2} \alpha_{n}-\beta_{n} \alpha_{2}} & \cdots & -\frac{\beta_{4} \alpha_{n}-\beta_{n} \alpha_{4}}{\beta_{2} \alpha_{n}-\beta_{n} \alpha_{2}} & -\frac{\beta_{3} \alpha_{n}-\beta_{n} \alpha_{3}}{\beta_{2} \alpha_{n}-\beta_{n} \alpha_{2}}
\end{array}\right)_{(n-2) \times(n-2)}
$$

By direct computation, we have

$$
T_{F, n}^{-1}=B\left(N^{-1} \oplus M^{-1}\right) A=\left(\begin{array}{cccccccccc}
\frac{F_{n}}{F_{n+1}} & -1 & 0 & 0 & 0 & \cdots & \cdots & \cdots & 0 & \frac{(-1)^{n}}{F_{n+1}} \\
1 & -1 & -1 & 0 & 0 & \cdots & \cdots & \cdots & 0 & 0 \\
0 & 1 & -1 & -1 & 0 & & & & & 0 \\
\vdots & \ddots & 1 & -1 & -1 & \ddots & & & & \vdots \\
\vdots & & \ddots & \ddots & \ddots & \ddots & \ddots & & & \vdots \\
\vdots & & & \ddots & \ddots & \ddots & \ddots & \ddots & & \vdots \\
\vdots & & & & \ddots & \ddots & \ddots & \ddots & \ddots & \vdots \\
\vdots & & & & & \ddots & 1 & -1 & -1 & 0 \\
0 & \cdots & \cdots & \cdots & \cdots & \cdots & 0 & 1 & -1 & -1 \\
-\frac{1}{F_{n+1}} & 0 & \cdots & \cdots & \cdots & \cdots & \cdots & 0 & 1 & \frac{F_{n}}{F_{n+1}}
\end{array}\right)_{n \times n}
$$

Remark 3. It is well known that if you divide $F_{n}$ by $F_{n+1}$, then these ratios get closer and closer to about 0.618, which is known to many people as the Golden Ratio, a number which has fascinated mathematicians, scientists and artists for centuries. Equation (14) can be appreciated in many different ways, and it is easy to see that top-left and bottom-right corner entries of $T_{F, n}^{-1}$ get closer and closer to the Golden Ratio. In fact, Toeplitz matrices, tridiagonal Toeplitz matrices with perturbed corner entries, the Fibonacci number, and the Golden Ratio are all connected by Equation (14). 


\subsection{Determinant and Inverse Matrix of a Fankel Matrix}

In this subsection, the determinant and the inverse of the Fankel matrix $H_{F, n}$ are studied.

Theorem 3. Let $H_{F, n}$ be an $n \times n$ Fankel matrix defined as in (5). Then $H_{F, n}$ is invertible and

$$
\operatorname{det} H_{F, n}=(-1)^{\frac{(n-1) n}{2}} F_{n+1} \text {, }
$$

where $F_{n+1}$ is the $(n+1)$ th Fibonacci number.

Proof. From (8), it follows that $\operatorname{det} H_{F, n}=\operatorname{det} \hat{I}_{n} \operatorname{det} T_{F, n}$. We obtain this conclusion by the fact that $\operatorname{det} \hat{I}_{n}=(-1)^{\frac{n(n-1)}{2}}$ and Theorem 1 .

Remark 4. This Theorem gives the relationship between the Fankel matrix and the Fibonacci number. From the standpoint of number theory, the $(n+1)$ th Fibonacci number can be expressed as the product of the determinant of an $n \times n$ Fankel matrix and a sign function.

Theorem 4. Let $H_{F, n}$ be an $n \times n$ Fankel matrix defined as in (5). Then inverse matrix of $H_{F, n}$ is

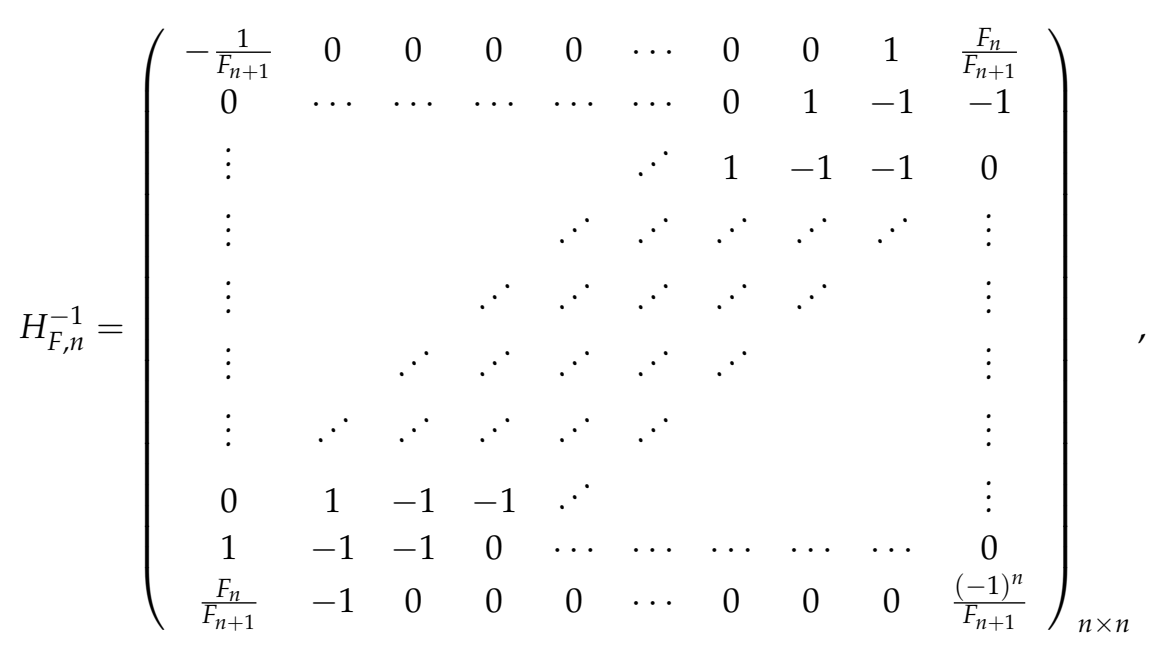

where $F_{n}$ and $F_{n+1}$ are the $n$th and $(n+1)$ th Fibonacci numbers, respectively.

Proof. We obtain this conclusion by formula (8) and Theorem 2.

Remark 5. Equation (16) can be appreciated in many different ways, and it is easy to see that bottom-left and top-right corner entries of $H_{F, n}^{-1}$ get closer and closer to the Golden Ratio. In fact, Hankel matrices, sub-tridiagonal Hankel matrices with perturbed corner entries, the Fibonacci number, and the Golden Ratio are all connected by Equation (16).

\subsection{Determinant and Inverse Matrix of a Loeplitz Matrix}

In this subsection, the determinant and the inverse of the Loeplitz matrix $T_{L, n}$ are studied.

Theorem 5. Let $T_{L, n}$ be an $n \times n$ Loeplitz matrix defined as in (6). Then $T_{L, n}$ is invertible and

$$
\operatorname{det} T_{L, n}=(-1)^{n+1} L_{n+1}-2^{n}, \text { for } n \geq 1 \text {, }
$$

where $L_{n+1}$ is the $(n+1)$ th Lucas number. 
Proof. For $n \leq 3$, it is easy to check that

$$
\operatorname{det} T_{L, 1}=1, \operatorname{det} T_{L, 2}=-8 \text { and } \operatorname{det} T_{L, 3}=-1 .
$$

Therefore, Equation (17) is satisfied. Now, we consider the case $n>3$. Define additional nonsingular matrices,

$$
\Delta_{1}=\left(\begin{array}{ccccccc}
1 & & & & & \\
-L_{-n} & & & & & 1 \\
-L_{-n-1} & & & & 1 & -1 \\
0 & & & 1 & -1 & -1 \\
\vdots & & . & . & . & . & \\
0 & 1 & -1 & -1 & &
\end{array}\right)_{n \times n}
$$

Multiplying $T_{L, n}$ by $\Delta_{1}$ from the left, we obtain

$$
\Delta_{1} T_{L, n}=\left(\begin{array}{ccccccc}
L_{1} & L_{2} & L_{3} & \cdots & L_{n-2} & L_{n-1} & L_{n} \\
0 & a_{2} & a_{3} & \cdots & a_{n-2} & a_{n-1} & a_{n} \\
0 & b_{2} & b_{3} & \cdots & b_{n-2} & b_{n-1} & b_{n} \\
0 & \cdots & \cdots & 0 & 2 & -1 & 0 \\
\vdots & & . & . & . & . & \vdots \\
\vdots & . & 2 & -1 & . & & \vdots \\
0 & 2 & -1 & 0 & \cdots & \cdots & 0
\end{array}\right)_{n \times n}
$$

where

$$
\left\{\begin{array}{l}
a_{i}=-L_{-n} L_{i}+L_{-n+i-1},(i=2,3, \cdots, n-1) \\
a_{n}=-L_{-n} L_{n}+L_{1} \\
b_{i}=-L_{-n-1} L_{i}+L_{-n+i-2},(i=2,3, \cdots, n-2) \\
b_{n-1}=-L_{-n-1} L_{n-1}+L_{1}-L_{-2} \\
b_{n}=-L_{-n-1} L_{n}+L_{2}-L_{1} .
\end{array}\right.
$$

Then, multiplying $\Delta_{1} T_{L, n}$ by $B_{1}$ from the right, we have

$$
\Delta_{1} T_{L, n} B_{1}=\left(\begin{array}{ccccccc}
L_{1} & L_{n} & L_{n-1} & L_{n-2} & \cdots & L_{3} & L_{2} \\
0 & a_{n} & a_{n-1} & a_{n-2} & \cdots & a_{3} & a_{2} \\
\vdots & b_{n} & b_{n-1} & b_{n-2} & \cdots & b_{3} & b_{2} \\
\vdots & 0 & -1 & 2 & 0 & \cdots & 0 \\
\vdots & \vdots & \ddots & \ddots & \ddots & \ddots & \vdots \\
\vdots & \vdots & & \ddots & -1 & 2 & 0 \\
0 & 0 & \cdots & \cdots & 0 & -1 & 2
\end{array}\right)_{n \times n},
$$


and

$$
\begin{aligned}
\operatorname{det}\left(\Delta_{1} T_{L, n} B_{1}\right)= & \operatorname{det}\left(\Delta_{1}\right) \operatorname{det}\left(T_{L, n}\right) \operatorname{det}\left(B_{1}\right) \\
= & L_{1}\left(a_{n} \sum_{i=1}^{n-2} 2^{n-2-i} b_{n-i}-b_{n} \sum_{i=1}^{n-2} 2^{n-2-i} a_{n-i}\right) \\
= & 2^{n-3}\left[\left(-L_{-n-1} L_{n-1}+L_{1}-L_{-2}\right)\left(-L_{-n} L_{n}+L_{1}\right)-\left(-L_{-n} L_{n-1}+L_{-2}\right)\right. \\
& \left.\cdot\left(-L_{-n-1} L_{n}+L_{0}\right)\right]+\sum_{i=2}^{n-2} 2^{n-2-i}\left[\left(-L_{-n-1} L_{n-i}+L_{-i-2}\right)\left(-L_{-n} L_{n}+L_{1}\right)\right. \\
& \left.-\left(-L_{-n} L_{n-i}+L_{-i-1}\right)\left(-L_{-n-1} L_{n}+L_{0}\right)\right] .
\end{aligned}
$$

From the definition of $\Delta_{1}$ and $B_{1}$, we get

$$
\operatorname{det} \Delta_{1}=\operatorname{det} B_{1}=(-1)^{\frac{(n-1)(n-2)}{2}} .
$$

By formulas (2) and (3), we obtain

$$
\operatorname{det} T_{L, n}=(-1)^{n+1} L_{n+1}-2^{n},
$$

which completes the proof.

Remark 6. This Theorem gives the relationship between the Loeplitz matrix and the Lucas number. From the perspective of number theory, the $(n+1)$ th Lucas number can be expressed as the sum of the determinant of $n \times n$ Loeplitz matrix and scalar matrix.

Theorem 6. Let $T_{L, n}$ be an $n \times n$ Loeplitz matrix defined as in (6). Then

$$
T_{L, 1}^{-1}=1, T_{L, 2}^{-1}=\left(\begin{array}{cc}
-\frac{1}{8} & \frac{3}{8} \\
\frac{3}{8} & -\frac{1}{8}
\end{array}\right), T_{L, 3}^{-1}=\left(\begin{array}{ccc}
8 & -9 & -5 \\
15 & -17 & -9 \\
-13 & 15 & 8
\end{array}\right),
$$

and for $n>3, T_{L, n}^{-1}$ is

$$
\mathbf{T}_{L, n}^{-1}=\left(\begin{array}{ccccccc}
Q_{3} & Q_{2} & 2^{n-3} Q_{1} & \cdots & 2^{2} Q_{1} & 2 Q_{1} & Q_{1} \\
Q_{4} & Q_{5} & Q_{2} & \ddots & \ddots & 2^{2} Q_{1} & 2 Q_{1} \\
2 Q_{4} & Q_{6} & Q_{5} & \ddots & \ddots & \ddots & 2^{2} Q_{1} \\
\vdots & 2 Q_{6} & \ddots & \ddots & \ddots & \ddots & \vdots \\
2^{n-4} Q_{4} & \vdots & \ddots & \ddots & Q_{5} & Q_{2} & 2^{n-3} Q_{1} \\
2^{n-3} Q_{4} & 2^{n-4} Q_{6} & \cdots & 2 Q_{6} & Q_{6} & Q_{5} & Q_{2} \\
Q_{7} & 2^{n-3} Q_{4} & 2^{n-4} Q_{4} & \cdots & 2 Q_{4} & Q_{4} & Q_{3}
\end{array}\right)_{n \times n}
$$


where

$$
\begin{aligned}
& Q_{1}=\frac{5}{\operatorname{det} T_{L, n}}, \\
& Q_{2}=1+2^{n-2} Q_{1}, \\
& Q_{3}=\frac{\operatorname{det} T_{L, n-1}}{\operatorname{det} T_{L, n}}, \\
& Q_{4}=\frac{(-1)^{n}\left(L_{n}+L_{n+2}\right)}{\operatorname{det} T_{L, n}}, \\
& Q_{5}=3+2^{n-1} Q_{1}, \\
& Q_{6}=5+2^{n} Q_{1}, \\
& Q_{7}=\frac{2^{n-2}\left[L_{n}+(-1)^{n+1} L_{n-1}\right]+(-1)^{n}}{\operatorname{det} T_{L, n}}, \\
& \operatorname{det} T_{L, n}=(-1)^{n+1} L_{n+1}-2^{n},
\end{aligned}
$$

and $L_{j}(j=1, \pm 2, \cdots, \pm n)$ is the $j$ th Lucas number.

Proof. For $n \leq 3$, it is easy to check that

$$
T_{L, 1}^{-1}=1, T_{L, 2}^{-1}=\left(\begin{array}{cc}
-\frac{1}{8} & \frac{3}{8} \\
\frac{3}{8} & -\frac{1}{8}
\end{array}\right), T_{L, 3}^{-1}=\left(\begin{array}{ccc}
8 & -9 & -5 \\
15 & -17 & -9 \\
-13 & 15 & 8
\end{array}\right)
$$

Now, we consider the case $n \geq 4$. The explicit expression of the inverse of the Loeplitz matrix can be found by use of Equation (20). Define additionally two nonsigular matrices.

$$
\Delta_{2}=\left(\begin{array}{ccccc}
1 & & & & \\
& 1 & & & \\
& -\frac{b_{n}}{a_{n}} & 1 & & \\
& & & \ddots & \\
& & & & 1
\end{array}\right)_{n \times n}
$$

and

$$
\nabla_{2}=\left(\begin{array}{cccccc}
1 & -\frac{L_{n}}{L_{1}} & \tau_{n-1} & \cdots & \tau_{3} & \tau_{2} \\
0 & 1 & -\frac{a_{n-1}}{a_{n}} & \cdots & -\frac{a_{3}}{a_{n}} & -\frac{a_{2}}{a_{n}} \\
\vdots & 0 & 1 & 0 & \cdots & 0 \\
\vdots & \vdots & \ddots & \ddots & \ddots & \vdots \\
\vdots & \vdots & & \ddots & \ddots & 0 \\
0 & 0 & \cdots & \cdots & 0 & 1
\end{array}\right)_{n \times n}
$$

where

$$
\tau_{i}=\frac{L_{n} a_{i}}{L_{1} a_{n}}-\frac{L_{i}}{L_{1}},(i=2,3, \cdots, n-1),
$$

with $a_{i}$ and $b_{i}$ are defined as in (18). 
Multiplying $\Delta_{1} T_{L, n} B_{1}$ by $\Delta_{2}$ from the left and by $\nabla_{2}$ from the right, we get

$$
\Delta T_{L, n} \nabla=\Delta_{2} \Delta_{1} T_{L, n} B_{1} \nabla_{2}=\left(\begin{array}{ccccccc}
L_{1} & 0 & 0 & 0 & \cdots & 0 & 0 \\
0 & a_{n} & 0 & 0 & \cdots & 0 & 0 \\
\vdots & 0 & \gamma_{n-1} & \gamma_{n-2} & \cdots & \gamma_{3} & \gamma_{2} \\
\vdots & 0 & -1 & 2 & 0 & \cdots & 0 \\
\vdots & \vdots & \ddots & \ddots & \ddots & \ddots & \vdots \\
\vdots & \vdots & & \ddots & \ddots & \ddots & 0 \\
0 & 0 & \cdots & \cdots & 0 & -1 & 2
\end{array}\right)_{n \times n},
$$

where

$$
\begin{aligned}
& \Delta=\Delta_{2} \Delta_{1}=\left(\begin{array}{ccccccc}
1 & & & & & 0 \\
-L_{-n} & & & & & 1 \\
\frac{b_{n} L_{-n}}{a_{n}}-L_{-n-1} & & & & 1 & \frac{-b_{n}}{a_{n}}-1 \\
0 & & & 1 & -1 & -1 \\
\vdots & & . \cdot & . & . & \\
0 & 1 & -1 & -1 & & &
\end{array}\right)_{n \times n}, \\
& \nabla=B_{1} \nabla_{2}=\left(\begin{array}{cccccc}
1 & -\frac{L_{n}}{L_{1}} & \tau_{n-1} & \cdots & \tau_{3} & \tau_{2} \\
0 & \cdots & \cdots & \ldots & 0 & 1 \\
\vdots & & & . & 1 & 0 \\
\vdots & & . & . & . & \vdots \\
\vdots & . & 1 & 0 & \ldots & 0 \\
0 & 1 & -\frac{a_{n-1}}{a_{n}} & \cdots & -\frac{a_{3}}{a_{n}} & -\frac{a_{2}}{a_{n}}
\end{array}\right)_{n \times n} \text {, } \\
& \gamma_{i}=-\frac{b_{n} a_{i}}{a_{n}}+b_{i},(i=2,3, \cdots, n-1),
\end{aligned}
$$

with $a_{i}, b_{i}$ and $\tau_{i}$ are defined as in (18) and (21), respectively. In addition, the matrix $\Delta T_{L, n} \nabla$ admits a block partition of the form

$$
\Delta T_{L, n} \nabla=\mathcal{N} \oplus \mathcal{M},
$$

where $\mathcal{N} \oplus \mathcal{M}$ denotes the direct sum of the matrices $\mathcal{N}$ and $\mathcal{N} . \mathcal{N}=\operatorname{diag}\left(L_{1}, a_{n}\right)$ is a nonsingular diagonal matrix,

$$
\mathcal{M}=\left(\begin{array}{cccccc}
\gamma_{n-1} & \gamma_{n-2} & \gamma_{n-3} & \cdots & \gamma_{3} & \gamma_{2} \\
-1 & 2 & 0 & \cdots & \cdots & 0 \\
0 & -1 & 2 & \ddots & & \vdots \\
\vdots & \ddots & \ddots & \ddots & \ddots & \vdots \\
\vdots & & \ddots & -1 & 2 & 0 \\
0 & \cdots & \cdots & 0 & -1 & 2
\end{array}\right)_{(n-2) \times(n-2)}
$$


Denote $\ell=\gamma_{n-1}-V C^{-1} U \neq 0$, where $V=\left(\gamma_{n-2} \gamma_{n-3} \cdots \gamma_{3} \gamma_{2}\right)_{1 \times(n-3)}$,

$$
C=\left(\begin{array}{ccccc}
2 & 0 & \cdots & \cdots & 0 \\
-1 & 2 & \ddots & & \vdots \\
0 & \ddots & \ddots & \ddots & \vdots \\
\vdots & \ddots & -1 & 2 & 0 \\
0 & \cdots & 0 & -1 & 2
\end{array}\right)_{(n-3) \times(n-3)}
$$

and $U=\left(\begin{array}{llll}-1 & 0 & \cdots & 0\end{array}\right)_{1 \times(n-3)}^{T}$. From (22), we obtain

$$
T_{L, n}^{-1}=\nabla\left(\mathcal{N}^{-1} \oplus \mathcal{M}^{-1}\right) \Delta
$$

Based on the definitions of $\mathcal{N}$ and $\mathcal{M}$, we have $\mathcal{N}^{-1}=\operatorname{diag}\left(L_{1}^{-1}, a_{n}^{-1}\right)$. By Lemma 1 , we get

$$
C^{-1}=\left(\begin{array}{ccccccc}
\omega_{1} & 0 & \cdots & \cdots & \cdots & \cdots & 0 \\
\omega_{2} & \omega_{1} & \ddots & & & & \vdots \\
\omega_{3} & \omega_{2} & \omega_{1} & \ddots & & & \vdots \\
\vdots & \ddots & \ddots & \ddots & \ddots & & \vdots \\
\omega_{n-5} & \ddots & \ddots & \ddots & \ddots & \ddots & \vdots \\
\omega_{n-4} & \omega_{n-5} & \ddots & \ddots & \omega_{2} & \omega_{1} & 0 \\
\omega_{n-3} & \omega_{n-4} & \omega_{n-5} & \cdots & \omega_{3} & \omega_{2} & \omega_{1}
\end{array}\right)_{(n-3) \times(n-3)},
$$

where

$$
\omega_{i}=\frac{1}{2^{i}}, 1 \leq i \leq n-3
$$

From Lemma 5 in [20], we have

$$
\mathcal{M}^{-1}=\left(\begin{array}{cc}
\frac{1}{\ell} & -\frac{1}{\ell} V C^{-1} \\
-\frac{1}{\ell} C^{-1} U & C^{-1}+\frac{1}{\ell} C^{-1} U V C^{-1}
\end{array}\right)_{(n-2) \times(n-2)}
$$

where

$$
\begin{aligned}
& V C^{-1}=\left(\hat{\eta}_{1}, \hat{\eta}_{2}, \cdots, \hat{\eta}_{n-3}\right), \\
& C^{-1}+\frac{1}{\ell} C^{-1} U V C^{-1}=\left[m_{i, j}^{\prime}\right]_{i, j=1}^{n-3}, \\
& \hat{\eta}_{i}=\sum_{j=1}^{n-2-i} \gamma_{n-1-j} \omega_{i}, 1 \leq i \leq n-3, \\
& m_{i, j}^{\prime}=\omega_{i-j+1}-\frac{\hat{\eta}_{j}}{\ell}, 1 \leq j \leq i \leq n-3, \\
& m_{i, j}^{\prime}=-\frac{\hat{\eta}_{j}}{\ell}, 1 \leq i<j \leq n-3 .
\end{aligned}
$$


Therefore, we get

$$
\mathcal{N}^{-1} \oplus \mathcal{M}^{-1}=\left(\begin{array}{ccccccc}
1 & 0 & 0 & \cdots & \cdots & \cdots & 0 \\
0 & \frac{1}{a_{n}} & 0 & \cdots & \cdots & \cdots & 0 \\
0 & 0 & \frac{1}{\ell} & -\frac{\hat{\eta}_{1}}{\ell} & -\frac{\hat{\eta}_{2}}{\ell} & \cdots & -\frac{\hat{\eta}_{n-3}}{\ell} \\
\vdots & \vdots & \frac{\omega_{1}}{\ell} & m_{1,1}^{\prime} & m_{1,2}^{\prime} & \cdots & m_{1, n-3}^{\prime} \\
\vdots & \vdots & \frac{\omega_{2}}{\ell} & m_{2,1}^{\prime} & m_{2,2}^{\prime} & \cdots & m_{2, n-3}^{\prime} \\
\vdots & \vdots & \vdots & \vdots & \vdots & \ddots & \vdots \\
0 & 0 & \frac{\omega_{n-3}}{\ell} & m_{n-3,1}^{\prime} & m_{n-3,2}^{\prime} & \cdots & m_{n-3, n-3}^{\prime}
\end{array}\right)_{n \times n}
$$

Multiplying $\mathcal{N}^{-1} \oplus \mathcal{M}^{-1}$ by $\Delta$ from the right, we obtain

$$
\left(\mathcal{N}^{-1} \oplus \mathcal{M}^{-1}\right) \Delta=\left(\begin{array}{ccccccc}
1 & 0 & \cdots & \cdots & \cdots & \cdots & 0 \\
\frac{-L_{-n}}{a_{n}} & 0 & \cdots & \cdots & \cdots & 0 & \frac{1}{a_{n}} \\
c_{1,1} & c_{1,2} & c_{1,3} & c_{1,4} & c_{1,5} & \cdots & c_{1, n} \\
c_{2,1} & c_{2,2} & c_{2,3} & c_{2,4} & c_{2,5} & \cdots & c_{2, n} \\
c_{3,1} & c_{3,2} & c_{3,3} & c_{3,4} & c_{3,5} & \cdots & c_{3, n} \\
\vdots & \vdots & \vdots & \vdots & \vdots & \ddots & \vdots \\
c_{n-2,1} & c_{n-2,2} & c_{n-2,3} & c_{n-2,4} & c_{n-2,5} & \cdots & c_{n-2, n}
\end{array}\right)_{n \times n}
$$

where

$$
\begin{aligned}
& c_{1,1}=\frac{1}{\ell}\left(\frac{b_{n} L_{-n}}{a_{n}}-L_{-(n+1)}\right), c_{i, 1}=\frac{\omega_{i-1}}{\ell}\left(\frac{b_{n} L_{-n}}{a_{n}}-L_{-(n+1)}\right), 2 \leq i \leq n-2, \\
& c_{1,2}=-\frac{\hat{\eta}_{n-3}}{\ell}, c_{i, 2}=m_{i-1, n-3}^{\prime}, 2 \leq i \leq n-2, \\
& c_{1,3}=-\frac{\hat{\eta}_{n-4}}{\ell}+\frac{\hat{\eta}_{n-3}}{\ell}, c_{i, 3}=m_{i-1, n-4}^{\prime}-m_{i-1, n-3}^{\prime}, 2 \leq i \leq n-2, \\
& c_{1, j}=-\frac{\hat{\eta}_{n-1-j}}{\ell}+\frac{\hat{\eta}_{n-j}}{\ell}+\frac{\hat{\eta}_{n+1-j}}{\ell}, 4 \leq j \leq n-1, \\
& c_{i, j}=m_{i-1, n-1-j}^{\prime}-m_{i-1, n-j}^{\prime}-m_{i-1, n+1-j}^{\prime}, 2 \leq i \leq n-2,4 \leq i \leq n-1, \\
& c_{1, n}=\frac{1}{\ell}\left(\frac{-b_{n}}{a_{n}}-1\right)+\frac{\hat{\eta}_{1}}{\ell}, c_{i, n}=\frac{\omega_{i-1}}{\ell}\left(\frac{-b_{n}}{a_{n}}-1\right)-m_{i-1,1}^{\prime}, 2 \leq i \leq n-2 .
\end{aligned}
$$


By formulas (2) and (3), we have

$$
T_{L, n}^{-1}=\nabla\left(\mathcal{N}^{-1} \oplus \mathcal{M}^{-1}\right) \Delta=\left(\begin{array}{ccccccc}
Q_{3} & Q_{2} & 2^{n-3} Q_{1} & \cdots & 2^{2} Q_{1} & 2 Q_{1} & Q_{1} \\
Q_{4} & Q_{5} & Q_{2} & \ddots & \ddots & 2^{2} Q_{1} & 2 Q_{1} \\
2 Q_{4} & Q_{6} & Q_{5} & \ddots & \ddots & \ddots & 2^{2} Q_{1} \\
\vdots & 2 Q_{6} & \ddots & \ddots & \ddots & \ddots & \vdots \\
2^{n-4} Q_{4} & \vdots & \ddots & \ddots & Q_{5} & Q_{2} & 2^{n-3} Q_{1} \\
2^{n-3} Q_{4} & 2^{n-4} Q_{6} & \cdots & 2 Q_{6} & Q_{6} & Q_{5} & Q_{2} \\
Q_{7} & 2^{n-3} Q_{4} & 2^{n-4} Q_{4} & \cdots & 2 Q_{4} & Q_{4} & Q_{3}
\end{array}\right)_{n \times n}
$$

where $Q_{i}(i=1,2, \cdots, 7)$ is the same as in Theorem 6 .

\subsection{Determinant and Inverse Matrix of a Lankel Matrix}

In this subsection, the determinant and the inverse of the Lankel matrix $H_{L, n}$ are studied.

Theorem 7. Let $H_{L, n}$ be an $n \times n$ Lankel matrix defined as in (7). Then $H_{L, n}$ is invertible and

$$
\operatorname{det} H_{L, n}=(-1)^{\frac{n(n-1)}{2}}\left[(-1)^{n+1} L_{n+1}-2^{n}\right] \text {, }
$$

where $L_{n+1}$ is the $(n+1)$ th Lucas number.

Proof. From formula (9), it follows that $\operatorname{det} H_{L, n}=\operatorname{det} \hat{I}_{n} \operatorname{det} T_{L, n}$. We obtain the desired conclusion by using det $\hat{I}_{n}=(-1)^{\frac{n(n-1)}{2}}$ and Theorem 5 .

Remark 7. This Theorem gives the relationship between the Lankel matrix and the Lucas number. In terms of number theory, the $(n+1)$ th Lucas number can be expressed as the sum of the determinant of $n \times n$ Lankel matrix and scalar matrix.

Theorem 8. Let $H_{L, n}$ be an $n \times n$ Lankel matrix defined as in (7). Then

$$
H_{L, 1}^{-1}=1, H_{L, 2}^{-1}=\left(\begin{array}{cc}
\frac{3}{8} & -\frac{1}{8} \\
-\frac{1}{8} & \frac{3}{8}
\end{array}\right), H_{L, 3}^{-1}=\left(\begin{array}{ccc}
-13 & 15 & 8 \\
15 & -17 & -9 \\
8 & -9 & -5
\end{array}\right)
$$

and for $n>3, H_{L, n}^{-1}$ is

$$
H_{L, n}^{-1}=\left(\begin{array}{ccccccc}
Q_{7} & 2^{n-3} Q_{4} & 2^{n-4} Q_{4} & \cdots & 2 Q_{4} & Q_{4} & Q_{3} \\
2^{n-3} Q_{4} & 2^{n-4} Q_{6} & \cdots & 2 Q_{6} & Q_{6} & Q_{5} & Q_{2} \\
2^{n-4} Q_{4} & \vdots & . \cdot & . \cdot & Q_{5} & Q_{2} & 2^{n-3} Q_{1} \\
\vdots & 2 Q_{6} & . \cdot & . \cdot & . \cdot & . \cdot & \vdots \\
2 Q_{4} & Q_{6} & Q_{5} & . \cdot & . \cdot & . \cdot & 2^{2} Q_{1} \\
Q_{4} & Q_{5} & Q_{2} & . & . \cdot & 2^{2} Q_{1} & 2 Q_{1} \\
Q_{3} & Q_{2} & 2^{n-3} Q_{1} & \cdots & 2^{2} Q_{1} & 2 Q_{1} & Q_{1}
\end{array}\right)_{n \times n}
$$

where $Q_{i}(i=1,2, \ldots, 7)$ is the same as in Theorem 6 .

Proof. By formula (9), we have $H_{L, n}^{-1}=\hat{I}_{n} T_{L, n}^{-1}$. Thus we get the desired conclusion from Theorem 6 . 


\section{Example}

In this section, an example demonstrates the method which was introduced above for the calculation of the determinant and inverse of the Foeplitz matrix and the Loeplitz matrix.

Example 1. Here we consider an $8 \times 8$ Foeplitz matrix:

$$
\mathbf{T}_{F, 8}=\left(\begin{array}{cccccccc}
1 & 1 & 2 & 3 & 5 & 8 & 13 & 21 \\
-1 & 1 & 1 & 2 & 3 & 5 & 8 & 13 \\
2 & -1 & 1 & 1 & 2 & 3 & 5 & 8 \\
-3 & 2 & -1 & 1 & 1 & 2 & 3 & 5 \\
5 & -3 & 2 & -1 & 1 & 1 & 2 & 3 \\
-8 & 5 & -3 & 2 & -1 & 1 & 1 & 2 \\
13 & -8 & 5 & -3 & 2 & -1 & 1 & 1 \\
-21 & 13 & -8 & 5 & -3 & 2 & -1 & 1
\end{array}\right)_{8 \times 8}
$$

From formula (10), we obtain

$$
\operatorname{det} T_{F, 8}=F_{9}=34 \text {. }
$$

As the inverse calculation, if we use the corresponding formulas in Theorems 2, we have $F_{8}=21, F_{9}=34$. So we get

$$
\mathbf{T}_{F, 8}^{-1}=\left(\begin{array}{cccccccc}
\frac{21}{34} & -1 & 0 & 0 & 0 & 0 & 0 & \frac{1}{34} \\
1 & -1 & -1 & 0 & 0 & 0 & 0 & 0 \\
0 & 1 & -1 & 1 & 0 & 0 & 0 & 0 \\
0 & 0 & 1 & -1 & -1 & 0 & 0 & 0 \\
0 & 0 & 0 & 1 & -1 & -1 & 0 & 0 \\
0 & 0 & 0 & 0 & 1 & -1 & -1 & 0 \\
0 & 0 & 0 & 0 & 0 & 1 & -1 & -1 \\
-\frac{1}{34} & 0 & 0 & 0 & 0 & 0 & 1 & \frac{21}{34}
\end{array}\right)_{8 \times 8}
$$

Example 2. Here we consider a $5 \times 5$ Loeplitz matrix:

$$
\mathbf{T}_{L, 5}=\left(\begin{array}{ccccc}
1 & 3 & 4 & 7 & 11 \\
3 & 1 & 3 & 4 & 7 \\
-4 & 3 & 1 & 3 & 4 \\
7 & -4 & 3 & 1 & 3 \\
-11 & 7 & -4 & 3 & 1
\end{array}\right)_{5 \times 5} .
$$

From formula (18), we obtain

$$
\operatorname{det} T_{L, 5}=-\left(2 L_{7}-L_{9}\right)-2^{5}=-14
$$


As the inverse calculation, if we use the corresponding formulas in Theorem 6 , we have $Q_{1}=-\frac{5}{14}, Q_{2}=$ $-\frac{13}{7}, Q_{3}=\frac{27}{14}, Q_{4}=\frac{20}{7}, Q_{5}=-\frac{19}{7}, Q_{6}=-\frac{45}{7}, Q_{7}=-\frac{143}{14}$. So we get

$$
\mathbf{T}_{L, 5}^{-1}=\left(\begin{array}{rrrrr}
\frac{27}{14} & -\frac{13}{7} & -\frac{10}{7} & -\frac{5}{7} & -\frac{5}{14} \\
\frac{20}{7} & -\frac{19}{7} & -\frac{13}{7} & -\frac{10}{7} & -\frac{5}{7} \\
\frac{40}{7} & -\frac{45}{7} & -\frac{19}{7} & -\frac{13}{7} & -\frac{10}{7} \\
\frac{80}{7} & -\frac{90}{7} & -\frac{45}{7} & -\frac{19}{7} & -\frac{13}{7} \\
-\frac{143}{14} & \frac{80}{7} & \frac{40}{7} & \frac{20}{7} & \frac{27}{14}
\end{array}\right)_{5 \times 5} .
$$

Author Contributions: Conceptualization, methodology, funding acquisition, Z.J.; writing-original draft preparation, W.W.; investigation, resources, formal analysis, software, B.Z. and B.N.; writing-review and editing, supervision, visualization, Y.Z.

Funding: The research was funded by the National Natural Science Foundation of China (Grant No. 11671187), the Natural Science Foundation of Shandong Province (Grant No. ZR2016AM14) and the PhD Research Foundation of Linyi University (Grant No. LYDX2018BS067), China.

Acknowledgments: The authors are grateful to the anonymous referees for their useful suggestions which improve the contents of this article.

Conflicts of Interest: The authors declare no conflict of interest.

\section{References}

1. Mukhexjee, B.N.; Maiti, S.S. On some properties of positive definite Toeplitz matrices and their possible applications. Linear Algebra Appl. 1988, 102, 211-240. [CrossRef]

2. Basilevsky, A. Applied Matrix Algebra in the Statistical Sciences; North Holland: New York, NY, USA, 1983.

3. Bai, Z.Z.; Li, G.Q.; Lu, L.Z. Combinative preconditioners of modified incomplete Cholesky factorization and Sherman-Morrison-Woodbury update for self-adjoint elliptic Dirichlet-periodic boundary value problems. J. Comput. Math. 2004, 22, 833-856.

4. Chen, H.; Lv, W.; Zhang, T.T. A Kronecker product splitting preconditioner for two-dimensional space-fractional diffusion equations. J. Comput. Phys. 2018, 360, 1-14. [CrossRef]

5. Chen, H.; Zhang, T.T.; Lv, W. Block preconditioning strategies for time-space fractional diffusion equations. Appl. Math. Comput. 2018, 337, 41-53. [CrossRef]

6. Chen, H.; Wang X.L.; Li X.L. A note on efficient preconditioner of implicit Runge-Kutta methods with application to fractional diffusion equations. Appl. Math. Comput. 2019, 351, 116-123. [CrossRef]

7. Grenander, U.; Szego, G. Toeplitz Forms and Their Applications; University of California Press: Berkeley, CA, USA, 1958.

8. Iohvidov, I.S. Hankel and Toeplitz Matrices and Forms (Algebruic Theory); Translated by G. Philips and A. Thijse; Birkhäuser: Boston, MA, USA, 1982.

9. Heinig, G.; Rost, K. Algebraic Methods for Toeplitz-Like Matrices and Operators; Oper. Theory 13; Birtiuser: Basel, Switzerland, 1984.

10. Zheng, Y.P.; Shon, S. Exact determinants and inverses of generalized Lucas skew circulant type matrices. Appl. Math. Comput. 2015, 270,105-113. [CrossRef]

11. Jiang, X.Y.; Hong, K.C. Explicit inverse matrices of Tribonacci skew circulant type matrices. Appl. Math. Comput. 2015, 268, 93-102. [CrossRef]

12. Bozkurt, D.; Tam, T.Y. Determinants and inverses of circulant matrices with Jacobsthal and Jacobsthal-Lucas Numbers. Appl. Math. Comput. 2012, 219, 544-551. [CrossRef]

13. Li, J.; Jiang, Z.L.; Lu, F.L. Determinants, norms, and the spread of circulant matrices with Tribonacci and generalized Lucas numbers. Abstr. Appl. Anal. 2014, 2014, 381829. [CrossRef] 
14. Jiang, Z.L.; Gong, Y.P.; Gao, Y. Invertibility and explicit inverses of circulant-type matrices with $k$-Fibonacci and $k$-Lucas numbers. Abstr. Appl. Anal. 2014, 2014, 238953.

15. Jiang, Z.L.; Gong, Y.P.; Gao, Y. Circulant type matrices with the sum and product of Fibonacci and Lucas numbers. Abstr. Appl. Anal. 2014, 2014, 375251. [CrossRef]

16. Jiang, X.Y.; Hong, K.C. Exact determinants of some special circulant matrices involving four kinds of famous numbers. Abstr. Appl. Anal. 2014, 2014, 273680. [CrossRef]

17. Akbulak, M.; Bozkurt, D. On the norms of Toeplitz matrices involving Fibonacci and Lucas numbers. Hacet. J. Math. Stat. 2008, 37, 89-95.

18. Thomas, K. Fibonacci and Lucas Numbers with Applications; John Wiley \& Sons: New York, NY, USA, 2001.

19. Zuo, B.S.; Jiang, Z.L.; Fu, D.Q. Determinants and inverses of Ppoeplitz and Ppankel matrices. Special Matrices 2018, 6, 201-215. [CrossRef]

20. Liu, L.; Jiang, Z.L. Explicit form of the inverse matrices of Tribonacci circulant type matrices. Abstr. Appl. Anal. 2015, 2015, 169726. [CrossRef]

(c) 2019 by the authors. Licensee MDPI, Basel, Switzerland. This article is an open access article distributed under the terms and conditions of the Creative Commons Attribution (CC BY) license (http:/ / creativecommons.org/licenses/by/4.0/). 